\title{
Bruxismo do sono
}

\author{
Cristiane Rufino de Macedo*
}

O bruxismo do sono é uma atividade oral caracterizada pelo ranger ou apertar dos dentes durante o sono e que, geralmente, está associada com despertares curtos com duração de 3 a 15 segundos, conhecidos como microdespertares ${ }^{2}$. Embora o termo bruxismo origine-se do grego brychein, que significa ranger de dentes, outros nomes têm sido usados para descrever este quadro: neurose do hábito oclusal, neuralgia traumática, bruxomania, friccionar-ranger de dentes, briquismo, apertamento e parafunção oral ${ }^{7,8}$.

O bruxismo do sono se diferencia do bruxismo diurno por envolver: distintos estados de consciência, isto é, sono e vigília; e diferentes estados fisiológicos com diferentes influências na excitabilidade oral motora ${ }^{3,4,11}$. Assim, o bruxismo diurno é caracterizado por uma atividade semivoluntária da mandíbula, de apertar os dentes enquanto o indivíduo se encontra acordado, onde geralmente não ocorre o ranger de dentes, e está relacionado a um tique ou hábito. Já o bruxismo do sono é uma atividade inconsciente de ranger ou apertar os dentes, com produção de sons, enquanto o indivíduo encontra-se dormindo. O bruxismo do sono também é chamado de bruxismo noturno, mas o termo mais apropriado é bruxismo do sono, pois o ranger de dentes pode também se desenvolver durante o sono diurno.

O bruxismo é classificado como primário ou secundário. O bruxismo primário, por ser idiopático, não está relacionado a nenhuma causa médica evidente, clínica ou psiquiátrica. Esta forma primária parece ser um distúrbio crônico persistente, com evolução a partir do seu aparecimento na infância ou adolescência para a idade adulta. Já o bruxismo secundário está associado com outros transtornos clínicos: neurológico, como na doença de Parkinson; psiquiátrico, nos casos de depressão; outros transtornos do sono, como a apnéia; e uso de drogas, como as anfetaminas ${ }^{3}$.

A prevalência exata do bruxismo do sono na população é imprecisa e subestimada. Isto ocorre porque os estudos epidemiológicos são baseados em populações e metodologias diferentes. Por exemplo, o relato de indivíduos que dormem sozinhos e não têm consciência dos sons produzidos durante o seu sono, pode ser diferente dos questionários preenchidos por portadores ou familiares com diferentes definições clínicas e diferente sintomatologia ${ }^{3}$. Embora existam estas limitações, estudos têm mostrado que a taxa de prevalência em crianças maiores de 11 anos de idade é a mais alta, variando entre $14 \%$ e $20 \%^{1}$. Nos adultos jovens, entre 18 e 29 anos de idade, é de 13\%, diminuindo ao longo da vida para $3 \%$ em indivíduos acima de 60 anos de idade ${ }^{16}$. A prevalência na população idosa deve ser maior que a estimada, já que as próteses totais em acrilico previnem os sons de ranger de dentes. Não tem sido encontrada diferença de gênero para a ocorrência do bruxismo do sono ${ }^{16}$.

Vários são os fatores de risco associados ao bruxismo do sono: idade, tabaco, álcool, cafeína,

* Mestre e doutoranda pela Universidade Federal de São Paulo (UNIFESP) e clínica geral particular. 
Fatores psicológicos, como estresse e personalidade, também são relacionados como fatores etiológicos ${ }^{3,15}$. Contudo alguns estudos falharam em demonstrar a relação entre o bruxismo do sono e o estresse ${ }^{21,23}$. Parece que os indivíduos com bruxismo do sono têm como característica serem focados em realizar atividades com um forte objetivo de alcançar o sucesso quando comparados aos indivíduos controles, em contraste a um distúrbio de ansiedade ou ao estresse patológico ${ }^{5}$. 


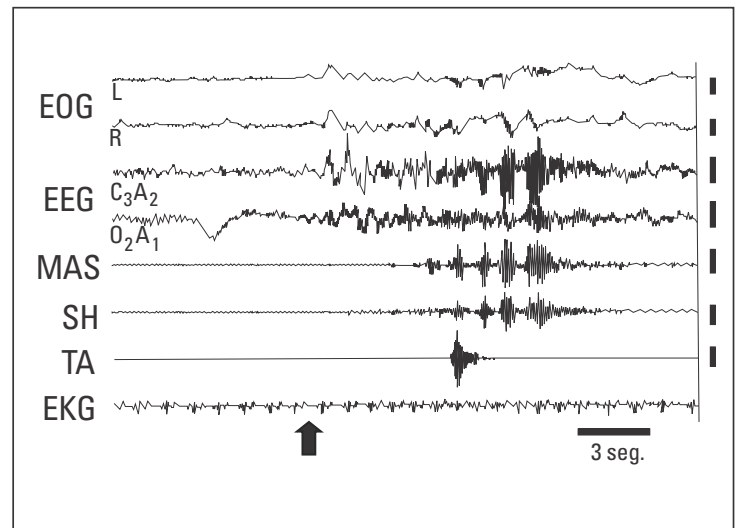

FIGURA 1 - Episódios de AMMR com ranger de dentes. Microdespertar (seta) com mudança na atividade do EEG $\left(C_{3} A_{2}\right.$ e $\left.0_{2} A_{1}\right)$, alteração ECG (EKG), seguida das contrações fásicas do masseter (MAS) e atividade EMG do suprahióide (SH) com ranger de dentes. Eletro-oculograma (EOG) e atividade muscular tibial anterior (TA). Fonte: Kato et al. ${ }^{6}$

com a primeira batida cardíaca 1 segundo antes do AMMR. Há o aumento do tônus do suprahióide 0,8 segundos antes do início, que provavelmente é o responsável pela abertura da mandíbula e da via aérea e, finalmente, ocorre a AMMR com a alteração do EMG nos músculos masseteres, com ou sem ranger de dentes ${ }^{12}$ (Fig. 1, 2).

A incidência do microdespertar está associada à alta freqüência da atividade muscular mastigatória. A magnitude dos episódios de AMMR - como início mais rápido da aceleração do ritmo cardíaco, maior atividade do EMG e uma maior força do contato dentário com ranger de dentes - é o que difere os indivíduos com bruxismo do sono dos indivíduos sem ${ }^{15}$.

Além dos microdespertares, há indícios da participação de substâncias neuroquímicas na gênese do bruxismo ${ }^{18}$. Em um ensaio clínico controlado, Lobbezoo et al. ${ }^{18}$ demonstraram, por meio de polissonografia, que o uso da levodopa (precursor da dopamina) resultou em diminuição significante, de cerca de 30\%, do bruxismo do sono. Ainda que não esteja claro o papel da serotonina na fisiopatologia do bruxismo, drogas que são inibidoras seletivas da receptação da serotonina - tais como a

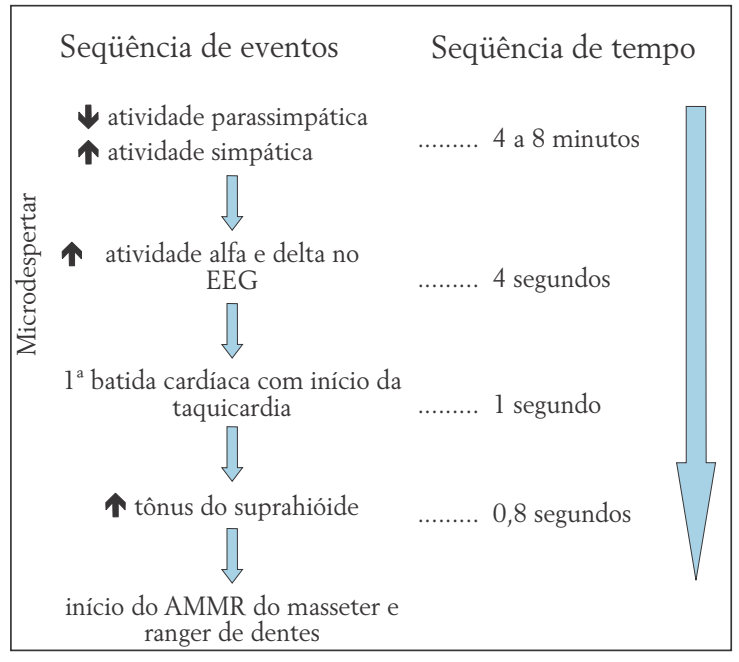

FIGURA 2 - Fluxograma dos eventos fisiológicos do bruxismo do sono.

fluoxetina, sertralina, paroxetina - têm sido apontadas como causadoras do ranger de dentes ${ }^{19}$.

Clinicamente, o diagnóstico é baseado no relato de ranger de dentes ocorrido durante o sono associado à dor ou tensão nos músculos da face ao acordar. O desgaste anormal dos dentes e a hipertrofia do masseter são sinais que ajudam no diagnóstico. Entretanto, a confiabilidade desses achados é duvidosa, pois o desgaste dentário pode ter ocorrido anteriormente ao evento do bruxismo e a hipertrofia pode ter sido secundária ao hábito de apertamento voluntário do indivíduo.

O diagnóstico clínico pode ser complementado pela polissonografia, que irá identificar os episódios de bruxismo durante a noite de sono (Fig. 3). É importante que esse exame tenha - além dos canais de eletroencefalograma, eletro-oculograma e eletromiografia - o registro audiovisual, para que seja observado o som de ranger de dentes e excluídas outras atividades bucomandibulares - tais como salivação, deglutição, mioclonias, tosse, vocalização - que representam mais de 30\% das atividades bucais durante o sono e que podem ser confundidas com os episódios de bruxismo do sono.

Atualmente, não existe nenhuma estratégia es- 


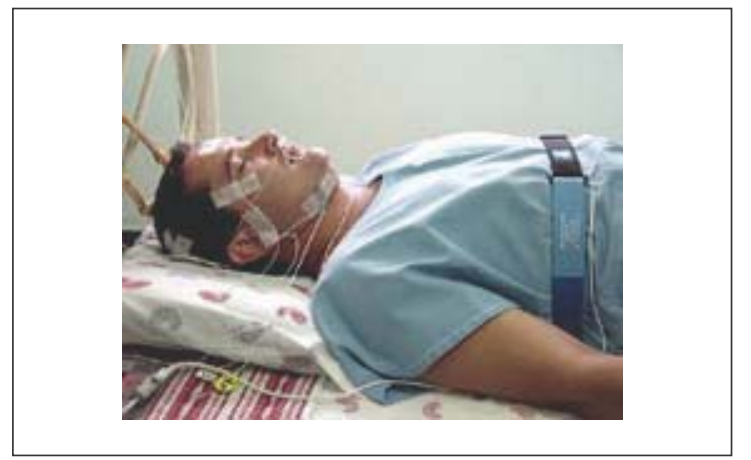

FIGURA 3 - Realização do exame de polissonografia durante o sono. Fonte: www. sinomar.com.br

pecífica, tratamento único ou cura para o bruxismo do sono. Diferentes linhas de tratamento têm sido propostas: tratamentos farmacológicos, psicológicos e odontológicos. O tratamento farmacológico é utilizado para casos agudos e graves e consiste no uso dos fármacos por um período curto de tempo. Drogas do tipo benzodiazepínicos, anticonvulsivantes, beta-bloqueadores, agentes dopaminérgicos, antidepressivos e relaxantes musculares são as mais utilizadas, mas não há nenhuma droga de primeira eleição. O tratamento psicológico consiste na terapia comportamental baseada na higiene do sono, no controle do estresse, biofeedback e em técnicas de relaxamento, mas nenhum desses tratamentos é baseado em fortes evidências. $\mathrm{O}$ tratamento odontológico para o bruxismo inclui ajuste oclusal, restauração da superfície dentária, tratamento ortodôntico e placas oclusais ${ }^{8}$.

Atualmente, o tratamento odontológico mais utilizado é o das placas oclusais. Nos EUA foi estimada a fabricação de 1 milhão e 200 mil placas oclusais por ano, a um custo de 275 dólares por placa, estima-se um total de 330 milhões de dólares gastos por ano na sua produção ${ }^{25}$. Alguns estudos associam o uso da placa oclusal com a diminuição da atividade EMG durante o sono, melhora na redução dos sintomas e prevenção do desgaste oclusal $^{10,24,26}$. Contrariamente, outros autores encontraram um aumento da atividade muscular em $20 \%$ para as placas oclusais rígidas e $50 \%$ para as flexíveis, e afirmam que a única ação das placas seria de proteção dos dentes ao desgaste ${ }^{5,22}$.

A efetividade das placas oclusais foi avaliada por meio de uma revisão sistemática Cochrane, que teve como objetivo identificar, analisar e comparar os dados de todos os estudos clínicos randomizados publicados e não publicados na literatura nacional e internacional, sem restrição de idiomas ${ }^{19}$. As placas oclusais foram comparadas com outras placas como palatal, TENS e ao não tratamento nos indivíduos com bruxismo do sono. Após o mapeamento completo do conhecimento, identificação e análise dos estudos, Macedo et al. ${ }^{19}$ concluíram que as evidências são insuficientes para afirmar que a placa oclusal é efetiva para o tratamento do bruxismo do sono e que a indicação ou não do seu uso é questionável quanto aos desfechos do sono (episódios de bruxismo, sons de ranger de dentes, microdespertar, despertar, eficiência do sono, tempo total de sono), mas pode ser que haja algum benefício para o desgaste dentário ${ }^{19}$.

A falta de evidências nos tratamentos para o bruxismo do sono não nos permite apenas contemplar esse distúrbio como algo que ainda precisa ser desvendando. Diante da falta de evidências quanto à efetividade dos tratamentos existentes para o bruxismo do sono, a decisão clínica do tratamento a ser realizado deve ser tomada em conjunto, baseada na experiência profissional aliada ao desejo do paciente.

Há necessidade de novos estudos para testar a efetividade dos tratamentos para o bruxismo do sono. O ensaio clínico randomizado é o desenho de estudo mais adequado para testar terapias que podem ser comparadas com outras. A metodologia rigorosa - como cálculo para determinação do tamanho da amostra, para aumentar o poder do estudo em estimar o efeito e a utilização dos guidelines, produzidos pelo grupo CONSORT pode melhorar a qualidade dos ensaios clínicos que proporcionarão novas respostas às nossas dúvidas sobre o bruxismo do sono ${ }^{20}$. 


\section{REFERÊNCIAS}

1. $A B E, K$; SHIMAKAWA, M. Genetic and developmental aspects of sleeptalking and teeth-grinding. Acta Paedopsychiatr. Basel, v. 33, no. 11, p. 339-344, Nov. 1966.

2. AMERICAN SLEEP DISORDERS ASSOCIATION. International classification of sleep disorder: diagnostic and coding manual, revised. 2nd ed. Westchester, 2005. p. 189-192.

3. BADER, G.; LAVIGNE, G. Sleep bruxism; an overview of an oromandibular sleep movement disorder. Sleep. Med. Rev., London v. 4, no. 1, p. 27-43, Feb. 2000.

4. CHASE, M. H.; MORALES, F. R. Control of motoeurons during sleep. In: KRYGER, M. H.; ROTH, T.; DEMENT, W. C. (Ed.). Principles and practice of sleep medicine. Philadephia: WB Saunders, 2000. p. 155-168.

5. DAO, T. T.; LUND, J. P.; LAVIGNE, G. J. Comparison of pain and quality of life in bruxers and patients with myofascial pain of the masticatory muscles. J. Orofac. Pain, Carol Stream, v. 8, no. 4, p. 350-356, Fall 1994.

6. DAO, T. T.; LAVIGNE, G. J. Oral splints: the crutches for temporomandibular disorders and bruxism? Crit. Rev. Oral Biol. Med., Alexandria, v. 9, no. 3, p. 345-361, 1998

7. DORLAND, W. A. Dorland's illustrated medical dictionary 29th ed. Philadelphia: W.B. Saunders, 2000

8. FAULKNER, K. B. D. Bruxism: a review of the literature. Part I. Aust. Dent. J., Sydney, v. 35, no. 3, p. 266-276, June 1990

9. GERBER, P. E.; LYND, L. D. Selective serotonin-reuptake inhibitorinduced movement disorders. Ann. Pharmacother, Cincinnati, v. 32, no. 6, p. 692-698, June 1998

10. HAMADA, T.; KOTANI, H.; KAWAZOE, Y.; YAMADA, $\mathrm{S}$. Effect of occlusal splints on the EMG activity of masseter and temporal muscles in bruxism with clinical symptoms. J. Oral Rehabil., Oxford, v. 9, no. 2, p. 119-123, Mar. 1982.

11. KATO, T.; MONTPLAISIR, J. Y.; GUITARD, F.; SESSLE, B. J.; LUND, J. P.; LAVIGNE, G. J. Evidence that experimentally induced sleep bruxism is a consequence of transient arousal. J. Dent. Res. Alexandria, v. 82, no. 4, p. 284-288, Apr. 2003

12. KATO, T.; ROMPRÉ, P. H.; MONTPLAISIR, J. Y.; SESSLE, B. J. LAVIGNE, G. J. Sleep bruxism: an oromotor activity secondary to micro-arousal. J. Dent. Res., Alexandria, v. 80, no. 10, p. 1940-1944, Oct. 2001

13. LAVIGNE, G. J. KATO, T. KOLTA, A.; SESSLE, B. J.

Neurobiological mechanisms involved in sleep bruxism. Crit. Rev. Oral Biol. Med., Alexandria, v. 14, no. 1, p. 30-46, 2003.

14. LAVIGNE, G. J.; LOBBEZOO, F.; ROMPRE, P. H.; NIELSEN, T. A. MONTPLAISIR, J. Y. Cigarette smoking as a risk factor or an exacerbating factor for restless legs syndrome and sleep bruxism. Sleep, Winchester, v. 20, no. 4, p. 290-293, Apr. 1997.
15. LAVIGNE, G. J.; MANZINE, C. Bruxism. In: KRYGER, M. H.; ROTH, T.; DEMENT, W. C. (Ed.). Principles and practice of sleep medicine. Philadelphia: W. B. Saunders, 2000. p. $773-785$.

16. LAVIGNE, G. J.; MONTPLAISIR, J. Y. Restless legs syndrome and sleep bruxism: prevalence and association among Canadians. Sleep, Winchester, v. 17, no. 8, p. 739-743, Dec. 1994

17. LAVIGNE, G. J.; ROMPRÉ, P. H.; POIRER, G.; HUARD, H.; KATO, T.; MONTPLAISIR, J. Y. Rhythmic mastigatory muscle activity during sleep in humans. J. Dent. Res., Alexandria, v. 80 , no. 2, p. 443-448, Feb. 2001

18. LOBBEZOO, F.; LAVIGNE, G. J.; TANGUAY, R.; MONTPLAISIR, J. Y. The effect of catecholamine precursor L-dopa on sleep bruxism: a controlled clinical trial. Mov. Disord., New York, v. 12, no. 1, p. 73-78, Jan. 1997

19. MACEDO, C. R.; SILVA, A. B.; MACHADO, M. A.; SACONATO, H.; PRADO, G. F. Oclusal splint for treating sleep bruxism (tooth grinding). Cochrane Database Syst. Rev., Oxford, v. 17 no. 4, p. 18-27, Oct. 2007.

20. MOHER, D.; SCHULZ, K. F.; ALTMAN, D. The CONSORT Statement: revised recommendations for improving the quality of reports of parallel-group randomized trials 2001. Explore, New York, v. 1, no. 1, p. 40-45, Jan. 2005

21. OHAYON, M. M.; LI, K. K.; GUILLEMINAULT, C. Risk factors for sleep bruxism in the general population. Chest, Northbrook, v. 119, no. 1, p. 53-61, Jan. 2001.

22. OKESON, J. P. The effects of hard and soft occlusal splints on nocturnal bruxism. J. Am. Dent. Assoc., Chicago, v. 114, no. 6, p. 788-791, June 1987

23. PIERCE, C. J.; CHRISMAN, K.; BENNETT, M. E.; CLOSE, J. M. Stress, anticipatory stress and psychologic measures related to sleep bruxism. J. Orofac. Pain, Carol Stream, v. 9, no. 1, p. 51-56, Winter 1995.

24. PIERCE, C. J.: GALE, E. N. A comparison of different treatments for nocturnal bruxism. J. Dent Res., Chicago, v. 67, no. 3, p. 597-601, Mar. 1988.

25. PIERCE, C. J.; WEYANT, R. J.; BLOCK, H. M.; NEMIR, D. C., Dental splint prescription patterns: a survey. J. Am. Dent. Assoc., Chicago, v. 126, no. 2, p. 248- 254 Feb. 1995.

26. SHEIKHOLESLAM, A.; HOLMGREN, K.; RIISE, C. A clinical and electromyographic study of the long-term effects of an occlusal splint on the temporal and masseter muscles in patients with functional disorders and nocturnal bruxism. J. Oral Rehabil., Oxford, v. 13, no. 2, p. 137-145, Mar. 1986.
Endereço para correspondência

Cristiane Rufino de Macedo

E-mail: crisrufa@bol.com.br 